\title{
Proper Orientations of Chordal Graphs
}

\author{
Julio Araujo ${ }^{1}$, Alexandre Cezar ${ }^{1}$, Carlos V.G.C. Lima ${ }^{2}$, \\ Vinicius F. dos Santos ${ }^{3}$, Ana Silva ${ }^{1}$ \\ ${ }^{1}$ ParGO, Departamento de Matemática - UFC, Fortaleza, Brazil \\ ${ }^{2}$ ParGO, Departamento de Computação - UFC, Fortaleza, Brazil \\ ${ }^{3}$ Departamento de Ciência da Computação - UFMG, Belo Horizonte, Brazil \\ \{julio, anasilva\}@mat.ufc.br, alexcezar@alu.ufc.br \\ gclima@lia.ufc.br, viniciussantos@dcc.ufmg.br
}

\begin{abstract}
An orientation $D$ of a graph $G=(V, E)$ is a digraph obtained from $G$ by replacing each edge by exactly one of the two possible arcs with the same end vertices. For each $v \in V(G)$, the indegree of $v$ in $D$, denoted by $d_{D}^{-}(v)$, is the number of arcs with head $v$ in $D$. An orientation $D$ of $G$ is proper if $d_{D}^{-}(u) \neq$ $d_{D}^{-}(v)$, for all $u v \in E(G)$. An orientation with maximum indegree at most $k$ is called a k-orientation. The proper orientation number of $G$, denoted by $\vec{\chi}(G)$, is the minimum integer $k$ such that $G$ admits a proper $k$-orientation. We prove that determining whether $\vec{\chi}(G) \leq k$ is NP-complete for chordal graphs of bounded diameter. We also present a tight upper bound for $\vec{\chi}(G)$ on split graphs and a linear-time algorithm for quasi-threshold graphs.
\end{abstract}

\section{Introduction}

In this work all graphs are considered to be simple and finite. An orientation $D$ of a graph $G=(V, E)$ is a digraph obtained from $G$ by replacing each edge by exactly one of the two possible arcs with the same endvertices. For each $v \in V(G)$, the indegree of $v$ in $D$, denoted by $d_{D}^{-}(v)$, is the number of arcs with head $v$ in $D$. We use the notation $d^{-}(v)$ when the orientation $D$ is clear from the context. An orientation $D$ of $G$ is proper if $d^{-}(u) \neq$ $d^{-}(v)$, for all $u v \in E(G)$. An orientation with maximum indegree at most $k$ is called a $k$-orientation. The proper orientation number of a graph $G$, denoted by $\vec{\chi}(G)$, is the minimum integer $k$ such that $G$ admits a proper $k$-orientation [Ahadi and Dehghan 2013].

By induction, one can prove that for any graph $G$ it is possible to obtain a proper $\Delta(G)$-orientation of $G$. Moreover, since every proper orientation of $G$ induces a proper vertex coloring of $G$ (starting with color zero), it follows that $\omega(G)-1 \leq \chi(G)-1 \leq \vec{\chi}(G) \leq \Delta(G)$. Determining the proper orientation number has been studied in some graph classes. In [Ahadi and Dehghan 2013], the authors study the problem on regular bipartite graphs and prove its NP-hardness for planar graphs and also for line graphs of regular graphs. An improvement on its hardness is given in [Araujo et al. 2015] for planar subcubic graphs and for planar bipartite graphs of maximum degree 5. The authors also show that the proper orientation number of trees is at most 4 and provide an upper bound for bipartite graphs. In [Araujo et al. 2016], the upper bound for trees is generalized by showing that the proper orientation number of cacti is at most 7 , which is tight. It is also proved that the tight upper bound for planar claw-free graphs is 6 . Other bounds for subclasses of planar graphs can be found 
in [Knox et al. 2017, Ai et al. ]. In [Araújo et al. 2019] the authors extend the notion of proper orientations for edge-weighted graphs. In this case the goal is similar by considering that the indegree of a vertex $v$ is given by the sum of the weights of its incident arcs whose heads are $v$. Other closely related weighted parameters have also been defined [Ahadi et al. 2017, Dehghan 2019].

Despite the number of results pointed out above, the study of proper orientations on dense graphs is quite scarce. The difficulty comes from the number of the possible orientations. In this work we study proper orientations on chordal graphs. We prove the NP-completeness of the problem of determining whether $\vec{\chi}(G) \leq k$, for a given chordal graph $G$ and positive integer $k$, even if the diameter of $G$ is bounded by a constant. With respect to the proper orientation number, we present a tight upper bound for split graphs and a linear-time algorithm to compute it for quasi-threshold graphs.

\section{Definitions}

Given disjoint vertex sets $A$ and $B$, we denote by $[A, B]$ the set of edges between $A$ and $B$. For $k \in \mathbb{N}$ we denote $[k]=\{1, \ldots, k\}$. The neighborhood of a vertex $v$ in a subgraph $H$ of $G$ is denoted by $N_{H}(v)$, that is, the set of neighbors of $v$ that are in $V(H)$. The degree of $v$ in $H$ is denoted as $d_{H}(v)$. We omit the subscript when it is clear from the context. Let $\omega(G)$ be the size of a largest clique of a graph $G$.

Given two graphs $G$ and $H$, the disjoint union, or simply union, of $G$ and $H$ is the graph $G \vee H$, such that $V(G \vee H)=V(G) \cup V(H)$ and $E(G \vee H)=E(G) \cup E(H)$. Moreover, the join of $G$ and $H$ is the graph $G \wedge H$, where $V(G \wedge H)=V(G) \cup V(H)$ and $E(G \wedge H)=E(G) \cup E(H) \cup\{u v \mid u \in V(G), v \in V(H)\}$.

A chordal graph is a graph in which every cycle of length at least 4 has a chord, that is, an edge joining two non-consecutive vertices of the cycle. A split graph is that one whose vertex set can be partitioned into a clique and an independent set. A quasithreshold graph is isomorphic to a $K_{1}$, or can be formed by a finite sequence of two operations: the disjoint union of two quasi-threshold graphs or the join of a new vertex and a quasi-threshold graph. Both split and quasi-threshold are subclasses of chordal graphs. The class of quasi-threshold graphs is in fact the intersection of cographs and interval graphs (a subclass of chordal graphs). Such graphs are also known as trivially perfect graphs and comparability graphs of trees [Jing-Ho et al. 1996].

\section{NP-completeness for Chordal Graphs}

Given a graph $G$ and a positive integer $k$, let Proper Orientation be the problem of determining whether $\vec{\chi}(G) \leq k$. We prove its NP-completeness for chordal graphs of bounded diameter. We first present a set of gadgets used in our reduction.

We start by providing a structure that prevents a given vertex $v$ from having indegree $i$, for some $i \in[k]$, for any proper $k$-orientation $D$ of a graph $G$. We build a gadget such that $v$ is adjacent to a vertex $u$ and we ensure $u$ to have indegree $i$ and that the edge $u v$ is oriented towards $u$, in any proper $k$-orientation of $G$. In order to build such structure, we define another gadget $S(k)$, containing vertices $v_{0}, \ldots, v_{k}$, such that we will ensure that $v_{j}$ must have indegree $j$, for each $j \in\{0\} \cup[k]$, in any proper $k$-orientation of $G$. 
Definition 1. Given a non-negative integer $k$, let $S(k)$ be constructed as follows. First add to $S(k)$ a clique $K$ on $k+1$ vertices $\left\{v_{0}, \ldots, v_{k}\right\}$. Then, for each $j \in\{0\} \cup[k-1]$, add to $S(k)$ a clique $K^{j}$ on $(k+1-j)$ vertices such that the only common vertex of $K^{j}$ with other vertices in $S(k)$ is $v_{j}$. Finally, add edges from $v_{j}$ to all vertices of $K^{\ell}$, for every $j \in\{0\} \cup[k-2]$ and for every $j+1 \leq \ell \leq k-1$.

Proposition 2. For an integer $k>0$, let $G$ be a graph having an $S(k)$ as induced subgraph and let $K$ be the $(k+1)$-clique $\left\{v_{0}, \ldots, v_{k}\right\}$, as in Definition 1. Then, in any proper $k$ orientation $D$ of $G$, we have that $d_{D}^{-}\left(v_{j}\right)=j$, for each $v_{j} \in K$. Moreover, for each $u v \in$ $E(G)$ with $v \in S(k)$ and $u \in V(G) \backslash V(S(k))$, we have that uv must be oriented towards $u$.

Let $G$ be a graph having an $S(k)$ as induced subgraph and a vertex $u \in V(G) \backslash V(S(k))$ that is adjacent to $v_{j}$, for some $j \in\{0\} \cup[k]$. Then, by Proposition 2, the indegree of $u$ must be distinct from $j$, however we also have that the edge $u v_{j}$ is oriented towards $u$. Thus, to each value of indegree we may want to forbid to $u$ by attaching it to vertices in (distinct copies of) $S(k)$, we have to add one unit to its indegree.

In order to forbid indegrees to some vertex $u$ without increasing its indegree, we can construct another simple structure that can be attached to $u$ so that it will have a neighbor $u^{\prime}$ with indegree $j$, however the edge $u u^{\prime}$ will necessarily be oriented towards $u^{\prime}$.

Definition 3. Given an integer $k>0$ and $i \in[k] \backslash\{1\}$, let $F(i, k)$ be the graph obtained from $S(k)$ by adding a vertex $u^{\prime}$, called the head of $F(i, k)$, such that $u^{\prime}$ is adjacent to the vertices $v_{1}, \ldots, v_{i-1}$ in the clique $K$ of $S(k)$.

We say that a graph $G$ has a pendant $F(i, k)$ at $u \in V(G)$ if $G$ has $F(i, k)$ as an induced subgraph and the only edge in $[V(F(i, k)), V(G) \backslash V(F(i, k))]$ is $u u^{\prime}$, where $u^{\prime}$ is the head of $F(i, k)$.

Proposition 4. Let $G$ be a graph having a pendant $F(i, k)$ at $u \in V(G)$, with $i \in[k] \backslash\{1\}$. Then, any proper $k$-orientation $D$ of $G$ satisfies $d_{D}^{-}\left(u^{\prime}\right)=i$ and $u u^{\prime}$ is oriented towards $u^{\prime}$.

Proposition 4 allows us to forbid indegree $d \in\{2, \ldots, k\}$ to a vertex $u$ having a pendant $F(d, k)$ attached to it. Thus, the above construction enables us to associate a list of indegrees $L: V(G) \rightarrow 2^{\{0\} \cup[k]}$, for each $v \in V(G)$, such that $v$ must have indegree in $L(v)$ in order to obtain a proper $k$-orientation of $G$. Note that Proposition 4 cannot be used to forbid the values 0 and 1 from appearing in the list of a vertex and $S(k)$ is chordal. Then, we can prove the NP-completeness of Proper Orientation for chordal graphs with bounded diameter by reducing the well-known Dominating Set problem on split graphs to it, which is NP-complete [Bertossi 1984].

Our reduction is based on a specific instance as in the reduction in [Bertossi 1984]. In order to obtain this instance, we consider the fact that Dominating Set is NP-complete for 4-regular planar graphs [Garey and Johnson 1979]. Then, combining this result with the construction in [Raman and Saurabh 2008] for the W[2]-hardness of Dominating Set for split graphs parameterized by the size of the solution, it follows that.

Lemma 5. Dominating SET is NP-complete even for split graphs, partitioned into a clique $\mathcal{K}$ and independent set $\mathcal{I}$, such that $d_{\mathcal{I}}(v)=d_{\mathcal{K}}(u)=5$, for every $v \in \mathcal{K}$ and $u \in \mathcal{I}$.

Theorem 6. Proper Orientation is NP-complete on chordal graphs of bounded diameter.

The idea to prove Theorem 6 is to construct from an instance of Lemma 5 an instance of PROPER ORIENTATION by attaching to the input split graph several gadgets as in Definition 1 and 3. We force each vertex of the independent set to be, in any proper 
$\left(k^{\prime}+3\right.$ )-orientation (for a suitable $\left.k^{\prime}\right)$, the head of at least one arc coming from a subset of at most $k$ vertices in the clique. Thus, a proper $\left(k^{\prime}+3\right)$-orientation shall exist in the new graph if, and only if, there is a dominating set with size at most $k$ in the given split graph.

\section{Bound for Split Graphs and Algorithm on Quasi-Threshold Graphs}

We obtain a tight upper bound for $\vec{\chi}(G)$ on split graphs.

Theorem 7. Let $G$ be a split graph. Then, $\vec{\chi}(G) \leq 2 \omega(G)-2$.

Theorem 8. For every positive integer $\omega$, there exists a split graph $G$ with $\omega(G)=\omega$ such that $\vec{\chi}(G)=2 \omega-2$.

The upper bound is obtained by a well-chosen proper orientation. The tight example is obtained by adding to a complete graph on $\omega$ vertices several independent sets having as neighbors the same subset of vertices of the $K_{\omega}$. The idea is to increase the average indegree in the clique in any proper orientation, which leads to the lower bound.

Theorem 9. Determining $\vec{\chi}(G)$ in a quasi-threshold graph $G$ can be done in linear time.

The idea to prove Theorem 9 is to apply induction to prove that $\vec{\chi}(G)=\omega(G)-1$.

\section{References}

Ahadi, A. and Dehghan, A. (2013). The complexity of the proper orientation number. Inform. Process. Lett., 113(19):799-803.

Ahadi, A., Dehghan, A., and Saghafian, M. (2017). Is there any polynomial upper bound for the universal labeling of graphs? J. Combin. Optim., 34(3):760-770.

Ai, J., Gerke, S., Gutin, G., Shi, Y., and Taoqiu, Z. Proper orientation number of trianglefree bridgeless outerplanar graphs. J. Graph Theor., to appear.

Araujo, J., Cohen, N., de Rezende, S. F., Havet, F., and Moura, P. F. (2015). On the proper orientation number of bipartite graphs. Theor. Comput. Sci., 566(0):59-75.

Araujo, J., Havet, F., Sales, C. L., and Silva, A. (2016). Proper orientation of cacti. Theor. Comput. Sci., 639:14-25.

Araújo, J., Sales, C. L., Sau, I., and Silva, A. (2019). Weighted proper orientations of trees and graphs of bounded treewidth. Theor. Comput. Sci., 771:39-48.

Bertossi, A. A. (1984). Dominating sets for split and bipartite graphs. Inform. Process. Lett., 19(1):37-40.

Dehghan, A. (2019). On the semi-proper orientations of graphs. CoRR, abs/1905.02867.

Garey, M. R. and Johnson, D. S. (1979). Computers and Intractability: A Guide to the Theory of NP-Completeness. W. H. Freeman \& Co., New York, NY, USA.

Jing-Ho, Y., Jer-Jeong, C., and Chang, G. J. (1996). Quasi-threshold graphs. Discrete Appl. Math., 69(3):247-255.

Knox, F., Matsumoto, N., de la Maza, S. G. H., Mohar, B., and Sales, C. L. (2017). Proper orientations of planar bipartite graphs. Graph. Combinator., 33:1189-1194.

Raman, V. and Saurabh, S. (2008). Short cycles make W-hard problems hard: FPT algorithms for W-hard problems in graphs with no short cycles. Algorithmica, 52(2):203225 . 